\begin{tabular}{ll}
\hline \hline $\begin{array}{l}\text { MINING AND METALLURGY INSTITUTE BOR } \\
\text { UDK: 622 }\end{array}$ & $\begin{array}{l}\text { ISSN: 2334-8836 (Štampano izdanje) } \\
\text { ISSN: 2406-1395 (Online) }\end{array}$ \\
\hline \hline & DOI:10.5937/MMEB1404125M \\
UDK: 628.336.4:66.094.8(045)=111 & ${\text { Radmila Markovic }{ }^{*}, \text { Vojka Gardic }^{*}, \text { Branimir Jugovic }^{* *}, \text { Marija Stevanovic }^{* * * *}}^{*}$
\end{tabular}

\title{
DEWATERING OF SLUDGE OBTAINED BY NEUTRALISATION FROM SULFURIC-ACID WASTE SOLUTIONS ${ }^{* * * * *}$
}

\begin{abstract}
The aim of this paper was to investigate the possibility of removing the liquid phase from the sludge using the mechanical dewatering methods. The sludge was obtained by neutralization of sulfuric acid waste solution from the process of copper electrolysis, using $\mathrm{IM} \mathrm{Ca}(\mathrm{OH})_{2}$ with and without addition of $\mathrm{FeCl}_{3}$ and $\mathrm{AlCl}_{3}$ as coagulants. Classical method of filtration by gravity and vacuum filtration method were used for tests of sludge dewatering process in the laboratory conditions. Solution volume, amount of sample, dewatering duration was measured during the experiments. The obtained results, presented in this paper, showed that using the vacuum filtration method, high of sludge sample was reduced for $75 \%$, which is almost three times higher value compared to the results obtained using the gravity filtration. Data relating to the time of filtration, using different methods, show that in application of vacuum filtration method the dewatering time is almost 200 times faster compared to the time dewatering with gravity filtration. The shortest settling time was registered for the sludge that was obtained by neutralization process without coagulants addition.
\end{abstract}

Keywords: dewatering, gravity filtration, vacuum filtration, sulfuric acid waste solution

\section{INTRODUCTION}

The basic requirements of many production plants is reducing the amount of waste solution and reducing the pollutants concentration to the statutory values. Characteristics of waste solution are the basic condition for the choice of appropriate technologies for their treatment. However, highly efficient processes of purification the different waste solutions lead to the formation of large quantities of sludge [1] and, consequently, to a series of prob- lems relating to dewatering and manipulation in order to its final storage [2]. The application of conductive and convective drying sludge processes require a large amount of thermal, so it is not economical to apply only specified thermal methods.

Moisture from the sludge could be very efficient removal by sludge dewatering process, namely, by removing free water without evaporation caused by thermal methods. The most commonly used devices and

\footnotetext{
* Mining and Metallurgy Institute Bor, Zeleni bulevar 35, 19210 Bor, Serbia, e-mail: radmila.markovic@irmbor.co.rs

** Institute of Technical Sciences of SASA, Knez, Mihailova 35/IV, Belgrade, Serbia

*** Thermal Power Plant "MORAVA”, Svilajnac, Serbia

**** This work is the result of the Project TR 37001: "The Impact of Mining Waste from RTB Bor on the Pollution of the Surrounding Water System with the Proposal of the Measures and Procedures for Reduction Harmful Effects on the Environment", funded by the Ministry of Education, Science and Technological Development of the Republic of Serbia
} 


\section{EXPERIMENTAL PART}

\subsection{Chemical characterization of waste solutions}

methods for mechanical separation of the solid from the liquid phase are: devices that are used for both filtration and natural evaporation, slow sand filters, frame and band filter presses, vacuum filters, centrifuges [3].

Addition of flocculants is often necessary to promote sludge particle agglomeration into larger particles or flocks prior aim to solid-water separation, usually by mechanical dewatering.

Before dewatering, it is often necessary to add flocculants in order to agglomeration of smaller particles of sludge into larger or forming flocs in order to allow faster filtration. Many chemical flocculants and coagulants, cationic polyacrylamides (CPAM), anionic polyacrylamides (APAM), polyaluminium chloride (PAC), ferric chloride $\left(\mathrm{FeCl}_{3}\right)$, aluminum chloride $\left(\mathrm{AlCl}_{3}\right)$, are widely used due to their characteristics for separating of solid and liquid phases [4-6]. The aim of this study was to investigate the possibility of mechanical dewatering of sludge obtained by neutralization of sulfuric acid waste solutions from obtaining the cathode copper. The influence of flocculants, the time of filtration, mass of sludge and volume of water after dewatering and other parameters to be controlled during vacuum filtration and gravity filtration. The obtained results will be used for the selection of methods for a more efficient sludge dewatering.

Concentrations of the components in the real waste solutions that will be used for experiments were detected using the chemical methods $\left(\mathrm{g} / \mathrm{dm}^{3}\right): \mathrm{Cu}=6.03, \mathrm{Ni}=0.61$, As $=0.51, \mathrm{Fe}=0.15, \mathrm{Cl}^{\mathrm{l}}=0.0608, \mathrm{Bi}=0.0448$, $\mathrm{Al}=0.0033, \mathrm{Zn}=0.0237, \mathrm{Sb}=0.0123$, $\mathrm{Mg}=0.0078, \mathrm{~Pb}=0.0029, \mathrm{Co}=0.0012$, $\mathrm{Mn}=0.0002, \mathrm{H}_{2} \mathrm{SO}_{4}=83.2826$. Atomic absorption spectrophotometer procedure (AAS), on apparatus Perkin-Elmer - 403, was used for analysis the content of the next elements: $\mathrm{Cu}, \mathrm{Ni}, \mathrm{As}, \mathrm{Fe}, \mathrm{Zn}, \mathrm{Pb}, \mathrm{Mn}$ and Cd. Optical Emission Spectrometer with Inductively Coupled Plasma (ICP-OES), on apparatus SPECTRO, was used for determining the content of $\mathrm{Co}, \mathrm{Sb}, \mathrm{Te}, \mathrm{Al}, \mathrm{Bi}$ and $\mathrm{Mg}$. Standard volumetric titration procedure was used for determining the $\mathrm{Cl}^{-}$ion content and electro-gravimetrical method was used for $\mathrm{Cu}$ analysis.

\subsection{Neutralization}

Neutralization of sulfuric-acid waste solutions witch chemical content, presented in part 2.1., was carried out adding $1 \mathrm{M}$ solution of $\mathrm{Ca}(\mathrm{OH})_{2}$ (sample 1), $1 \mathrm{M}$ solution of $\mathrm{Ca}(\mathrm{OH})_{2}$ with adding of $\mathrm{FeCl}_{3}$ (sample 2) and $1 \mathrm{M}$ solution of $\mathrm{Ca}(\mathrm{OH})_{2}$ with adding of $\mathrm{AlCl}_{3}$ (sample 3). Neutralization process was carried out at the ambient temperature with intensively stirring up to $\mathrm{pH}$ value of 7.4 (Table 1).

Table 1 Chemical characterization of sludge obtained by neutralization process

\begin{tabular}{|c|c|r|r|r|}
\hline Element & Measuring unit & \multicolumn{1}{|c|}{ Sample 1 } & \multicolumn{1}{c|}{ Sample 2 } & \multicolumn{1}{c|}{ Sample 3 } \\
\hline $\mathrm{Cu}$ & $\%$ & 3.84 & 3.50 & 3.63 \\
\hline $\mathrm{As}$ & $\%$ & 0.51 & 0.64 & 0.64 \\
\hline $\mathrm{Ni}$ & $\%$ & 0.33 & 0.30 & 0.31 \\
\hline $\mathrm{Fe}$ & $\%$ & 0.13 & 0.26 & 0.17 \\
\hline $\mathrm{Zn}$ & $\%$ & 0.02 & 0.018 & 0.019 \\
\hline $\mathrm{Sb}$ & $\%$ & 0.013 & 0.015 & 0.015 \\
\hline $\mathrm{Bi}$ & $\%$ & 0.0037 & 0.0041 & 0.0037 \\
\hline $\mathrm{Pb}$ & $\%$ & $<0.01$ & $<0.01$ & $<0.01$ \\
\hline
\end{tabular}


Atomic absorption spectrophotometer procedure (AAS), on apparatus PerkinElmer - 403, was used for analyzing the content of the following elements: $\mathrm{Pb}, \mathrm{Ni}$, $\mathrm{Zn}$ and Fe. Optical emission spectrometer with inductively coupled plasma (ICP-OES), on apparatus ARL 340+ Plasma Vision, was used for determining the content of $\mathrm{Bi}, \mathrm{Sb}$ and As. Electro-gravimetrical method was used for determining the $\mathrm{Cu}$ content.

\subsection{Sludge dewatering}

The mass of dry sample of $5 \mathrm{~g}$ with chemical composition, presented in Table 1, was used for further experiments. The sample was transferred to a measuring cylinder of $50 \mathrm{ml}$ that was used for all other tests. The leveling and volume of this sample was measured. After adding the volume of $40 \mathrm{ml}$ bidistilled water and manual stirring during $5 \mathrm{~min}$, the level and volume of suspension was measured. Then, water was added to the ratio solid: liquid $=1$ : 8 . During the settling in graduated cylinder, the level of clear zone in the interval of $1 \mathrm{~min}$ was measured. Gravity and vacuum filtration was used for solid/liquid separation. Filter paper with low ash content marked as Quantitative Ashless, 100 circles 5A, $125 \mathrm{~mm}$, was used during the gravity filtration. Glass filter crucible, marked G3, was used for vacuum filtration. In a both cases, the filtrate volume was measured in measuring bottle.

\section{RESULTS AND DISCUSION}

\subsection{Gravity filtration}

The results for sludge dewatering, obtained by neutralization process of waste sulfuric-acid solutions with $1 \mathrm{M}$ solution of $\mathrm{Ca}(\mathrm{OH})_{2}$, with and without adding of $\mathrm{FeCl}_{3}$ and $\mathrm{AlCl}_{3}$, are presented in Table 2.

Table 2 Gravity filtration

\begin{tabular}{|c|c|c|c|c|c|c|c|c|}
\hline \multirow{3}{*}{ Sample } & $\begin{array}{c}\text { Mass of } \\
\text { dry } \\
\text { sample }\end{array}$ & $\begin{array}{c}\text { DM } \\
\text { water } \\
\text { volume }\end{array}$ & $\begin{array}{l}\text { Volume } \\
\text { of dry } \\
\text { sample }\end{array}$ & $\begin{array}{c}\text { Volume } \\
\text { of } \\
\text { suspense }\end{array}$ & $\begin{array}{l}\text { Settling } \\
\text { time }\end{array}$ & $\begin{array}{c}\text { Filtra- } \\
\text { tion } \\
\text { duration }\end{array}$ & $\begin{array}{l}\text { Filtrate } \\
\text { volume }\end{array}$ & $\begin{array}{c}\text { Mass } \\
\text { of wet } \\
\text { sample }\end{array}$ \\
\hline & \multicolumn{8}{|c|}{ Measuring unit } \\
\hline & g & ml & $\mathbf{m l}$ & ml & $\min$ & $\min$ & ml & $\mathrm{g}$ \\
\hline $1 / \mathrm{g}$ & 5 & 40 & 3 & 43 & 18 & 25 & 24 & 15.52 \\
\hline $2 / \mathrm{g}$ & 5 & 40 & 3 & 43 & 25 & 38 & 21 & 17.48 \\
\hline $3 / \mathrm{g}$ & 5 & 40 & 3 & 43 & 25 & 37 & 22.5 & 16.50 \\
\hline
\end{tabular}

The measured values during the gravity filtration are the same for all samples (Table 2). Mass of wet sample is higher of about 3-3.5 times compared to the mass of dry sample. The results obtained by dewatering of samples with addition of different coagulants in neutralization process showed that the settling ability of these samples (samples $2 / \mathrm{g}$ and $3 / \mathrm{g}$ ) is less than for the sample obtained without the addition of coagulants (sample 1/g).

\subsection{Vacuum filtration}

The second group of experiments was related to the vacuum filtration of the suspension through a glass filter crucible, porosity G3, diameter $125 \mathrm{~mm}$. Results are shown in Table 3. 
Table 3 Vacuum filtration

\begin{tabular}{|c|c|c|c|c|c|c|c|c|}
\hline \multirow{3}{*}{ Sample } & $\begin{array}{c}\text { Mass of } \\
\text { dry } \\
\text { sample }\end{array}$ & $\begin{array}{c}\text { DM } \\
\text { water } \\
\text { volume }\end{array}$ & $\begin{array}{c}\text { Volume } \\
\text { of dry } \\
\text { sample }\end{array}$ & $\begin{array}{c}\text { Volume } \\
\text { of } \\
\text { suspense }\end{array}$ & $\begin{array}{c}\text { Settling } \\
\text { time }\end{array}$ & $\begin{array}{c}\text { Filtration } \\
\text { duration }\end{array}$ & $\begin{array}{c}\text { Filtrate } \\
\text { volume }\end{array}$ & $\begin{array}{c}\text { Mass } \\
\text { of wet } \\
\text { sample }\end{array}$ \\
\cline { 2 - 11 } & \multicolumn{7}{|c|}{ Measuring unit } \\
\hline $1 / \mathrm{g}$ & $\mathbf{g}$ & $\mathbf{m l}$ & $\mathbf{m l}$ & $\mathbf{s}$ & $\mathbf{s}$ & $\mathbf{m l}$ & $\mathbf{g}$ \\
\hline $2 / \mathrm{v}$ & 5 & 40 & 3 & 43 & 15 & 8 & 38.6 & 7.62 \\
\hline $3 / \mathrm{v}$ & 5 & 40 & 3 & 43 & 15.2 & 9 & 38.2 & 8.18 \\
\hline
\end{tabular}

As in the classical filtration by gravity, the measurement results for different samples have the approximate values. The mass of sludge sample after filtration is 1.52-1.62 times higher than the mass of dry sample. The results obtained by dewatering of sample with different coagulants, added during the neutralization process, showed that the settling ability of these samples (samples $2 / \mathrm{g}$ and $3 / \mathrm{g}$ ) is less than settling ability of sample obtained without the coagulants addition (sample $1 / \mathrm{g})$.
Changing the sludge level after dewatering by classical methods of filtration by gravity and vacuum filtration method are presented on Figure 1. The results showed that higher change in sample level is registered for the sludge that was obtained by neutralization of metallurgical waste water with the addition of $\mathrm{AlCl}_{3}$ as coagulant (samples 3/g and 3/c). For sample marked $3 / \mathrm{g}$, the reduction was achieved in the sample level of $22.92 \%$ and for sample marked $3 / \mathrm{h}$, the reduction degree of sample level was $75 \%$.

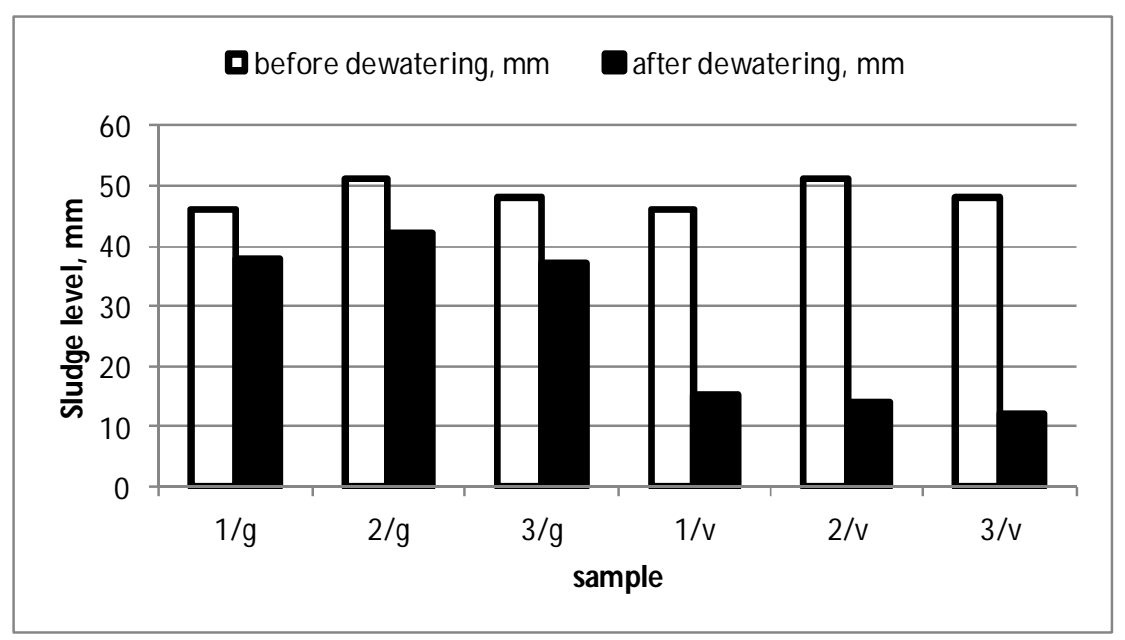

Figure 1 Changing the sludge level after dewatering by different filtration methods

Comparing the results for the content of the liquid phase in the individual sludge samples after filtration, classical and vacuum (Figure 2), the lowest percentage of liquid phase is registered in the samples $1 / \mathrm{g}$ and $1 / \mathrm{v}$ (sample obtained by neutralization process without adding of coagulants). 


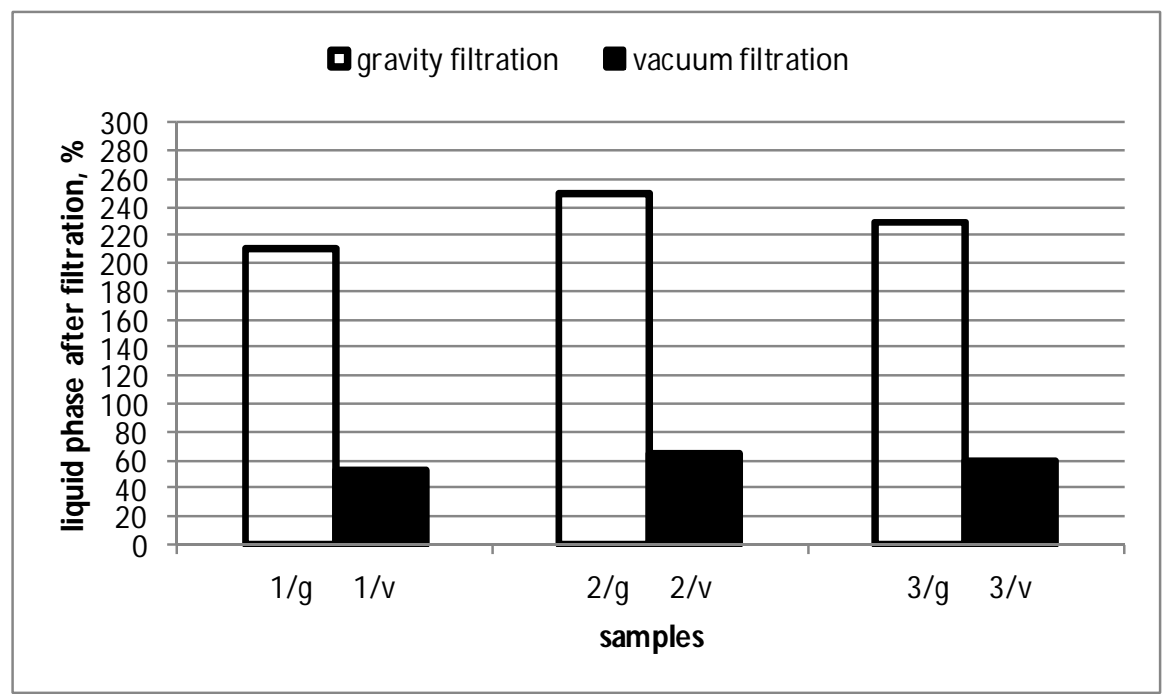

Figure 2 Dependence the liquid phase content of the sample type sample and used filtration type

The obtained results showed that the application of vacuum filtration gives better results for settling and better compacting of sludge.

\section{CONCLUSION}

The aim of this paper was to investigate dewatering sludge degree. The sludge was produced by neutralization process of waste sulfuric-acid solutions from cathode copper production with and without adding of coagulants. Classical methods of filtration by gravity and vacuum filtration were used for dewatering. The results showed that duration of vacuum filtration is much shorter than duration of conventional filtration. Volume of the liquid phase in sample after filtration was lowest in the sludge samples, obtained neutralizing without coagulants, and these values were $10.52 \mathrm{ml}$ and $2.62 \mathrm{ml}$ for samples marked $1 / \mathrm{g}$ and $1 / \mathrm{v}$, respectively. The results of vacuum filtration of different samples have achieved minor deviations in the values of measured parameters compared with the results of classical filtration.

\section{REFERENCES}

[1] Neyens, E.; Baeyens, J.; Dewil, R.; Heyder, B. D, Advanced Sludge Treatment Affects Extracellular Polymeric Substances to Improve Activated Sludge Dewatering, J. Haz. Mater. (2004) 106 (2-3): pp. 83-92.

[2] Irenem.C.Lo, Keithc. K. Lai and G.H. Chen, Salinity Effect on Mechanical Dewatering of Sludge with and without Chemical Conditioning, Environ. Sci. Technol. (2001) 35, 46914696.

[3] Lawrence K. Wang, Yan Li, Nazih K. Shammas and George P. Sakellaropoulos, Handbook of Environmental Engineering, Volume 6: Biosolids Treatment Processes, 13 Drying Beds (2007), 403-430, Edited by: L. K. Wang et al. (C) The Humana Press Inc., Totowa, NJ. 
[4] Huaili Zheng, Yongjun Sun, *Jinsong Guo, Fengting Li, Wei Fan, Yong Liao and Qingqing Guan, Characterization and Evaluation of Dewatering Properties of PADB, a Highly Efficient Cationic Flocculant, Ind. Eng. Chem. Res. 2014, 53, 2572-2582.

[5] Zhu, J. R.; Zheng, H. L.; Jiang, Z. Z.; Zhang, Z.; Liu, L. W.; Sun, Y. J.; Tshukudu, T. Synthesis and characterization of a dewatering reagent:
Cationic polyacrylamide (P(AMDMC-DAC)) for activated sludge dewatering treatment, Desalin. Water Treat. (2013) 51, 2791- 2801.

[6] Zheng, H. L.; Sun, Y. J.; Zhu, C. J.; Guo, J. S.; Zhao, C.; Liao, Y.; Guan, Q. Q. UV-initiated polymerization of hydrophobically associating cationic flocculants: Synthesis, characterization, and dewatering properties, Chem. Eng. J. (2013) 234, 318-326. 


\begin{tabular}{ll}
\hline \hline INSTITUT ZA RUDARSTVO I METALURGIJU BOR & ISSN: 2334-8836 (Štampano izdanje) \\
UDK: 622 & ISSN: 2406-1395 (Online) \\
\hline \hline
\end{tabular}

Radmila Marković, Vojka Gardić, Branimir Jugović, ${ }^{* *}$,Marija Stevanović****

\section{ODVODNJAVANJE MULJA DOBIJENOG NEUTRALIZACIJOM SUMPORNO-KISELIH OTPADNIH RASTVORA****}

Izvod

Cilj ovog rada bio je da se ispita mogućnost uklanjanja tečne faze iz mulja metodama mehaničkog odvodnjavanja. Mulj je dobijen neutralizacijom sumporno-kiselih otpadnih rastvora iz procesa elektrolize bakra, korišćenjem $1 \mathrm{M} \mathrm{Ca}(\mathrm{OH})_{2}$ sa i bez dodataka $\mathrm{FeCl}_{3}$ i $\mathrm{AlCl}_{3}$ kao koagulanata. Metoda klasične filtracije pod uticajem gravitacije i metoda vakuumske filtracije, korišćene su za ispitivanja procesa odvodnjavanja mulja u laboratorijskim uslovima. Tokom eksperimenata merena je zapremina rastvora, visina uzoraka, trajanje odvodnjavanja. Dobijeni rezultati prikazani u ovom radu pokazali su da je vakuumskom filtracijom postignuto smanjenje visine mulja od $75 \%$ što predstavlja gotovo 3 puta veću vrednost u odnosu na rezultate dobijene klasičnom filtracijom. Podaci koji se odnose na vreme filtracije primenom različitih metoda, pokazuju da je primenom vakuumske filtracione metode vreme odvodnjavanja gotovo 200 puta kraće u odnosu na vreme odvodnjavanja klasičnom filtracijom. Najkraće vreme bistrenja suspenzije registrovano je kod mulja dobijenog procesom neutralizacije bez. dodataka koagulanata.

Ključne reči: odvodnjavanje, klasična filtracija pod uticajem gravitacije, vakuumska filtracija, sumporno-kiseli otpadni rastvor

\section{UVOD}

Smanjenje količina otpadnih rastvora i koncentracije zagađujućih materija do zakonom propisanih vrednosti, su osnovni zahtevi mnogih proizvodnih postrojenja. Karakteristike otpadnih rastvora predstavljaju osnovni uslov za izbor odgovarjuće tehnologije za njihovo prečišćavanje. Međutim, visoko efikasni procesi prečišćavanja otpadnih rastvora različitog porekla dovode do nastajanja velikih količina mulja [1] i posledično, do niza problema koji se odnose na odvodnjavanje i manipulaciju u cilju njegovog krajnjeg skladištenja [2]. Primena konduktivnih i konvektivnih postupaka sušenja mulja zahteva veliku količinu toplotne energije, pa nije ekonomično primeniti samo navedene termičke metode. Vlaga iz mulja se vrlo efikasno uklanja i procesom odvodnjavanja mulja, odnosno uklanjanjem vode bez isparavanja izazvanog termičkim

\footnotetext{
* Institut za rudarstvo i metalurgiju Bor, Zeleni bulevar 35, 19210 Bor, Srbija,

e-mail: radmila.markovic@irmbor.co.rs

** Institut tehničkih nauka Srpske Akademije Nauka i Umetnosti, Knez Mihailova 35/IV, Beograd, Srbija

**** Termoelektrana "MORAVA", Svilajnac, Srbija

**** Ovaj rad je rezultat Projekta br TR: 37001 „Uticaj rudarskog otpada iz RTB Bor na zagađenje vodotokova, sa predlogom mera i postupaka za smanjenje štetnog dejstva na životnu sredinu“, finansiranog od strane Ministarstva prosvete, nauke i tehnološkog razvoja Republike Srbije
} 


\section{EKSPERIMENTALNI DEO}

\subsection{Hemijska karakterizacija otpadnih rastvora}

metodama. Najčešće korišćeni uređaji i metode za mehaničko razdvajanje čvrste od tečne faze su: uređaji u kojima se vrši istovremeno filtracija i prirodno isparavanje, spori peščani filtri, ramske i trakaste filter prese, vakuum filtri, centrifuge [3].

Pre odvodnjavanja, vrlo često je neophodno dodavanje flokulanata u cilju aglomeracije sitnijih čestica mulja u krupnije ili formiranja flokula u cilju omogućavanja lake filtracije. Mnogi hemijski flokulanti i koagulanti, katjonski poliakrilamidi (CPAM), anjonski poliakrilamidi (APAM), polialu-minijum hloridi (PAC), feri hloridi $\left(\mathrm{FeCl}_{3}\right)$, aluminijum hloridi $\left(\mathrm{AlCl}_{3}\right)$, u širokoj su upo-trebi zahvaljujući svojim osobinama za razdvajanje čvrste i tečne faze [4-6]. Cilj ovog rada bio je da se ispita mogućnost mehaničkog odvodnjavanja mulja dobijenog neutralizacijom otpadnih sumporno-kiselih rastvora iz procesa dobijanja katodnog bakra. Uticaj flokulanata, vreme filtracije, masa mulja i zapremina vode nakon odvodnjavanja i drugi parametri biće kontrolisani tokom vakuumske filtracije i klasične filtracije pod uticajem gravitacije. Dobijeni rezultati poslužiće za izbor metoda za što efikasnije odvodnjavanje mulja.
Hemijskom karakterizacijom realnih otpadnih rastvora koji će biti korišćeni za ispitivanja određene su koncentracije prisutnih komponenti $\left(\mathrm{g} / \mathrm{dm}^{3}\right)$ : $\mathrm{Cu}=6.03, \mathrm{Ni}=0.61$, $\mathrm{As}=0.51, \mathrm{Fe}=0.15, \mathrm{Cl}^{-}=0.0608, \mathrm{Bi}=0.0448$, $\mathrm{Al}=0.0033, \mathrm{Zn}=0.0237, \mathrm{Sb}=0.0123, \quad \mathrm{Mg}=$ $0.0078, \mathrm{~Pb}=0.0029, \mathrm{Co}=0.0012, \mathrm{Mn}=0.0002$, $\mathrm{H}_{2} \mathrm{SO}_{4}=83.2826$. Atomskom absorpcionom spektrofotometrijom (AAS), na aparatu Perkin-Elmer - 403 analizirani su: $\mathrm{Cu}, \mathrm{Ni}, \mathrm{As}$, $\mathrm{Fe}, \mathrm{Zn}, \mathrm{Pb}, \mathrm{Mn}$ i Cd. Optička emisiona spektrometrija sa indukovano kuplovanom plazmom (ICP-OES), na aparatu marke SPECTRO, korišćena je za određivanje sadržaja Co, Sb, Te, Al, Bi i Mg, Standardna metoda titracije korišćena je za određivanje sadržaja $\mathrm{H}_{2} \mathrm{SO}_{4}$, a turbidimetrijska metoda za određivanje sadržaja $\mathrm{Cl}^{-}$jona.

\subsection{Neutralizacija}

Neutralizacija otpadnog sumporno-kise$\log$ rastvora čija je hemijska karakterizacija prikazana u delu 2.1., rađena je sa $1 \mathrm{M}$ rastvorom $\mathrm{Ca}(\mathrm{OH})_{2}$ (uzorak 1 ), $1 \mathrm{M}$ rastvorom $\mathrm{Ca}(\mathrm{OH})_{2}$ uz dodatak $\mathrm{FeCl}_{3}$ (uzorak 2) i $1 \mathrm{M}$ rastvorom $\mathrm{Ca}(\mathrm{OH})_{2}$ uz dodatak $\mathrm{AlCl}_{3}$ (uzorak 3). Neutralizacija je rađena na sobnoj temperaturi, uz intenzivno mešanje do postizanja $\mathrm{pH}$ vrednosti od 7.4. (Tabela 1).

Tabela 1. Hemijska karakterizacija mulja dobijenog neutralizacijom

\begin{tabular}{|c|c|r|r|r|}
\hline Element & Jedinica mere & Uzorak 1 & \multicolumn{1}{c|}{ Uzorak 2 } & \multicolumn{1}{c|}{ Uzorak 3 } \\
\hline $\mathrm{Cu}$ & $\%$ & 3.84 & 3.50 & 3.63 \\
\hline $\mathrm{As}$ & $\%$ & 0.51 & 0.64 & 0.64 \\
\hline $\mathrm{Ni}$ & $\%$ & 0.33 & 0.30 & 0.31 \\
\hline $\mathrm{Fe}$ & $\%$ & 0.13 & 0.26 & 0.17 \\
\hline $\mathrm{Zn}$ & $\%$ & 0.02 & 0.018 & 0.019 \\
\hline $\mathrm{Sb}$ & $\%$ & 0.013 & 0.015 & 0.015 \\
\hline $\mathrm{Bi}$ & $\%$ & 0.0037 & 0.0041 & 0.0037 \\
\hline $\mathrm{Pb}$ & $\%$ & $<0.01$ & $<0.01$ & $<0.01$ \\
\hline
\end{tabular}


Atomska absorpciona spektrofotometrija (AAS), na opremi Perkin - Elmer - 403 korišćena je za određivanje sadržaja sledećih elemenata: $\mathrm{Pb}, \mathrm{Ni}, \mathrm{Zn}$ i Fe. Optička emisiona spektrometrija sa indukovano kuplovanom plazmom (ICP-OES), na aparatu marke ARL 340+ Plasma Vision, korišćena je za određivanje sadržaja $\mathrm{Bi}, \mathrm{Sb}$ i As, a elektrogravimetrijska metoda za određivanje sadržaja $\mathrm{Cu}$.

\subsection{Odvodnjavanje mulja}

Suvi uzorak mulja mase $5 \mathrm{~g}$, čiji je hemijski sastav prikazan u tabeli 1 , korišćen je za dalja ispitivanja. Uzorak je prebačen $u$ menzuru zapremine $50 \mathrm{ml}$ koja je korišćena za sva dalja merenja. Merena je zapremina i visina suvog uzorka, a nakon dodavanja 40 $\mathrm{ml}$ bidestilovane vode i ručnog mešanja $u$ trajanju od 5 min, merena je visina i zapremina suspenzije. Tokom bistrenja, odnosno taloženja čestica u graduisanom sudu, mere- na je visina zone bistre tečnosti u razmacima od po $1 \mathrm{~min}$. Klasična filtracija pod uticajem gravitacije i vakuumska filtracija korišćene su za razdvajanje faza. Filter papir sa niskim sadržajem pepela, oznake Quantitative Ashless, 100 otvora 5A, 125 mm, korišćen je tokom klasične filtracije pod uticajem gravitacije, a vakuum nuč filter, poroznosti G3, $125 \mathrm{~mm}$, za vakuumsku filtraciju. U oba slučaja merena je zapremina filtrata koji se sakupi za određeno vreme u baždarenoj boci.

\section{REZULTATI I DISKUSIJA}

\subsection{Klasična filtracija pod uticajem gravitacije}

Dobijeni rezultati odvodnjavanja mulja dobijenog neutralizacijom otpadnih sumporno - kiselih rastvora sa $1 \mathrm{M}$ rastvorom $\mathrm{Ca}(\mathrm{OH})_{2}$, sa i bez dodataka $\mathrm{FeCl}_{3}$ i $\mathrm{AlCl}_{3}$ prikazani su u tabeli 2 .

Tabela 2. Klasična filtracija pod uticajem gravitacije

\begin{tabular}{|c|c|c|c|c|c|c|c|c|}
\hline \multirow{3}{*}{ Uzorak } & $\begin{array}{c}\text { Masa } \\
\text { suvog } \\
\text { uzorka }\end{array}$ & $\begin{array}{c}\text { Zaprem. } \\
\text { DM vode }\end{array}$ & $\begin{array}{c}\text { Zaprem. } \\
\text { suvog } \\
\text { uzorka }\end{array}$ & $\begin{array}{c}\text { Zaprem. } \\
\text { suspenz. }\end{array}$ & $\begin{array}{c}\text { Vreme } \\
\text { bistrenja }\end{array}$ & $\begin{array}{c}\text { Vreme } \\
\text { filtrac. }\end{array}$ & $\begin{array}{c}\text { Zaprem. } \\
\text { filtrata }\end{array}$ & $\begin{array}{c}\text { Masa } \\
\text { uzorka } \\
\text { nakon } \\
\text { filtrac. }\end{array}$ \\
\cline { 2 - 10 } & \multicolumn{7}{|c|}{ Jedinica mere } \\
\cline { 2 - 10 } & $\mathrm{g}$ & $\mathrm{ml}$ & $\mathrm{ml}$ & $\mathrm{ml}$ & $\mathrm{min}$ & $\mathrm{min}$ & $\mathrm{ml}$ & $\mathrm{g}$ \\
\hline $1 / \mathrm{g}$ & 5 & 40 & 3 & 43 & 18 & 25 & 24 & 15.52 \\
\hline $2 / \mathrm{g}$ & 5 & 40 & 3 & 43 & 25 & 38 & 21 & 17.48 \\
\hline $3 / \mathrm{g}$ & 5 & 40 & 3 & 43 & 25 & 37 & 22.5 & 16.50 \\
\hline
\end{tabular}

Iz tabele 2 se vidi da su vrednosti dobijene merenjem tokom odvodnjavanja tri različita uzorka mulja, veoma bliske. Masa uzorka mulja nakon filtriranja je 3-3.5 puta veća od mase suvog uzorka. Rezultati dobijeni odvodnjavanjem mulja kod koga su dodati različiti koagulanti tokom procesa neutralizacije, pokazali su da je sposobnost taloženja ovih uzoraka (uzorci: 2/g i 3/g) manja nego uzorka dobijenog bez dodatka koagulanata (uzorak: 1/g).

\subsection{Vakuumska filtracija}

Druga grupa eksperimenata odnosila se na vakuumsku filtraciju suspenzije kroz stakleni nuč filter, poroznosti G3, prečnika 125 mm. Rezultati merenja prikazani su u tabeli 3. 
Tabela 3. Vakuumska filtracija

\begin{tabular}{|c|c|c|c|c|c|c|c|c|c|}
\hline \multirow{2}{*}{ Uzorak } & $\begin{array}{c}\text { Masa } \\
\text { Suvog } \\
\text { uzorka }\end{array}$ & $\begin{array}{c}\text { Zaprem. } \\
\text { DM vode }\end{array}$ & $\begin{array}{c}\text { Zaprem. } \\
\text { suvog } \\
\text { uzorka }\end{array}$ & $\begin{array}{c}\text { Zaprem. } \\
\text { suspenz. }\end{array}$ & $\begin{array}{c}\text { Vreme } \\
\text { bistrenja }\end{array}$ & $\begin{array}{c}\text { Vreme } \\
\text { filtrac. }\end{array}$ & $\begin{array}{c}\text { Zaprem. } \\
\text { filtrata }\end{array}$ & $\begin{array}{c}\text { Masa } \\
\text { uzorka } \\
\text { nakon } \\
\text { filtracije }\end{array}$ \\
\cline { 2 - 10 } & \multicolumn{7}{|c|}{ Jedinica mere } \\
\hline $\mathbf{1 / v}$ & 5 & $\mathrm{ml}$ & $\mathrm{ml}$ & $\mathrm{ml}$ & $\mathrm{s}$ & $\mathrm{s}$ & $\mathrm{ml}$ & $\mathrm{g}$ \\
\hline $\mathbf{2 / v}$ & 5 & 40 & 3 & 43 & 15 & 8 & 38.6 & 7.62 \\
\hline $\mathbf{3 / v}$ & 5 & 40 & 3 & 43 & 15.2 & 9 & 38.2 & 8.18 \\
\hline
\end{tabular}

Kao i prilikom klasične filtracije pod uticajem gravitacije, rezultati merenja za različite uzorke su približnih vrednosti. Masa uzorka mulja nakon filtriranja je 1.521.62 puta veća od mase suvog uzorka. RezuItati dobijeni odvodnjavanjem mulja kod koga su dodati različiti koagulanti tokom procesa neutralizacije, pokazali su da je sposobnost taloženja ovih uzoraka (uzorci: 2/g i 3/g) manja nego uzorka dobijenog bez dodatka koagulanata (uzorak: 1/g).
Na slici 1, prikazana je promena visine mulja nakon odvodnjavanja primenom metode klasične filtracije pod uticajem gravitacije i metode vakuumske filtracije. Rezultati su pokazali da je najveća promena postignuta kod uzorka mulja dobijenog neutralizacijom otpadne metalurške vode uz dodatak $\mathrm{AlCl}_{3}$ kao koagulanta (uzorci 3/g i $3 / \mathrm{v}$ ). Kod uzorka $3 / \mathrm{g}$ postignut je stepen smanjenja visine uzorka od $22.92 \%$, a kod uzorka 3/v smanjenje iznosi $75 \%$.

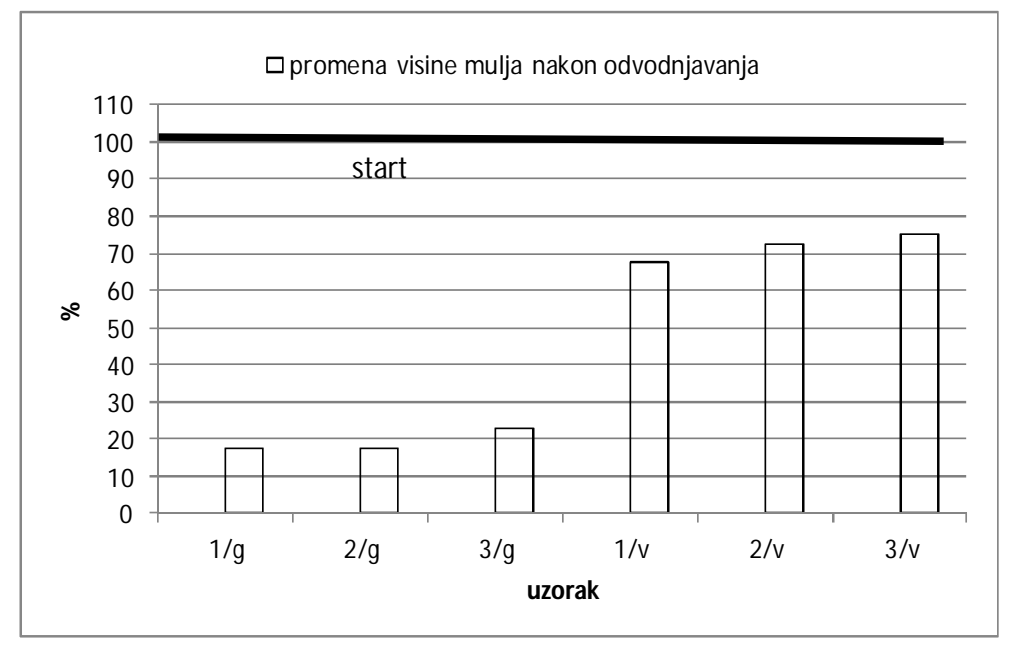

Sl. 1. Promena visine mulja nakon odvodnjavanja različitim metodama filtracije

Poređenjem rezultata za sadržaj tečne faze u pojedinačnim uzorcima mulja nakon filtracije, klasične i vakuumske, (slika 2), najmanji procenat tečne faze registrovan je $u$ uzorcima $1 / \mathrm{g}$ i $1 / \mathrm{v}$ (uzorak dobijen neutralizacijom bez dodataka koagulanata). 


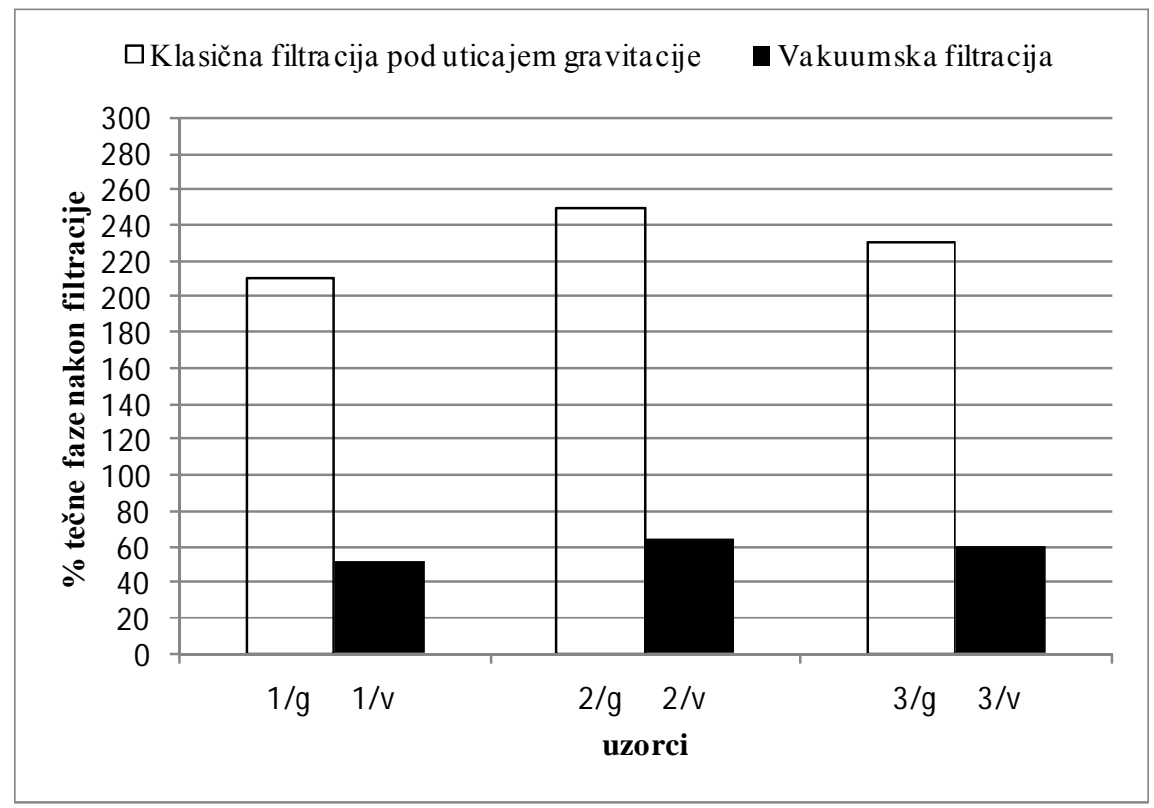

S1. 2. Zavisnost sadržaja tečne faze od vrste uzorka i korišćenog metoda filtracije

Rezultati su pokazali da primena vakuumske filtracije daje bolje rezultate kako kod odvodnjavanja tako i kod ugušćavanja ispitivanih uzoraka.

\section{ZAKLJUČAK}

Cilj ovog rada bio je ispitivanje stepena odvodnjavanja mulja dobijenog neutralizacijom otpadnih sumporno kiselih rastvora iz procesa proizvodnje katodnog bakra sa i bez dodataka kolagulanata. Za odvodnjavanje su korišćene metoda klasične filtracije pod uticajem gravitacije i metoda vakuumske filtracije. Rezultati su pokazali da je vreme vakuumske filtracije neuporedivo kraće od vremena klasične filtracije. Zapremina tečne faze u uzorku nakon filtriranja bila je najmanja $\mathrm{u}$ uzorcima mulja dobijenim neutralizacijom bez dodataka koagulanata i iznosila je $10.52 \mathrm{ml}$ i $2.62 \mathrm{ml}$ kod uzoraka $1 / \mathrm{g}$ i $1 / \mathrm{v}$, respektivno. Vakuumskom filtracijom različitih uzoraka postignuta su manja odstupanja u vrednostima merenih parame- tara $\mathrm{u}$ poređenju sa rezultatima klasične filtracije.

\section{LITERATURA}

[1] Neyens, E.; Baeyens, J.; Dewil, R.; Heyder, B. D, Advanced sludge treatment affects extracellular polymeric substances to improve activated sludge dewatering, J. Haz. Mater. (2004) 106 (2-3): 83-92.

[2] Irenem.C. Lo, Keithc. K. Lai and G. H. Chen, Salinity Effect on Mechanical Dewatering of Sludge with and without Chemical Conditioning, Environ. Sci. Technol. (2001) 35, 4691-4696.

[3] Lawrence K. Wang, Yan Li, Nazih K. Shammas and George P. Sakellaropoulos, Handbook of Environmental Engineering, Volume 6: Biosolids Treatment Processes, 13 Drying Beds (2007), 403-430, Edited by: L. K. Wang et al. (C) The Humana Press Inc., Totowa, NJ. 
[4] Huaili Zheng, Yongjun Sun,*Jinsong Guo, Fengting Li, Wei Fan, Yong Liao and Qingqing Guan, Characterization and Evaluation of Dewatering Properties of PADB, a Highly Efficient Cationic Flocculant, Ind. Eng. Chem. Res. 2014, 53, 2572-2582.

[5] Zhu, J. R.; Zheng, H. L.; Jiang, Z. Z.; Zhang, Z.; Liu, L. W.; Sun, Y. J.; Tshukudu, T. Synthesis and characterization of a dewatering reagent:
Cationic polyacrylamide (P(AMDMC-DAC)) for activated sludge dewatering treatment, Desalin. Water Treat. (2013) 51, 2791- 2801.

[6] Zheng, H. L.; Sun, Y. J.; Zhu, C. J.; Guo, J. S.; Zhao, C.; Liao, Y.; Guan, Q. Q. UV-initiated polymerization of hydrophobically associating cationic flocculants: Synthesis, characterization, and dewatering properties, Chem. Eng. J. (2013) 234, 318-326. 